\title{
Ferromagnetic nanorings
}

\author{
C A F Vaz ${ }^{1,9}$, T J Hayward ${ }^{1}$, J Llandro ${ }^{1}$, F Schackert $^{1}$, D Morecroft ${ }^{1}$, \\ J A C Bland ${ }^{1}$, M Kläui' ${ }^{2}$, M Laufenberg ${ }^{2}$, D Backes ${ }^{2,3}$, U Rüdiger ${ }^{2}$, \\ F J Castaño ${ }^{4}$, C A Ross ${ }^{4}$, L J Heyderman ${ }^{3}$, F Nolting ${ }^{5}$, A Locatelli ${ }^{6}$, \\ G Faini $^{7}$, S Cherif ${ }^{8}$ and $W$ Wernsdorfer $^{8}$ \\ ${ }^{1}$ Cavendish Laboratory, Madingley Road, Cambridge CB3 0HE, UK \\ ${ }^{2}$ Fachbereich Physik, Universität Konstanz, Universitätsstrasse 10, 78457 Konstanz, Germany \\ ${ }^{3}$ Laboratory for Micro- and Nanotechnology, Paul Scherrer Institut, Villigen PSI, Switzerland \\ ${ }^{4}$ Department of Materials Science and Engineering, Massachusetts Institute of Technology, \\ Cambridge, MA 02139, USA \\ ${ }^{5}$ Swiss Light Source, Paul Scherrer Institut, Villigen PSI, Switzerland \\ ${ }^{6}$ Sincrotrone Trieste, 34012 Basovizza, Trieste, Italy \\ ${ }^{7}$ Laboratoire de Photonique et de Nanostructures - CNRS, Route de Nozay, 91460 Marcoussis, \\ France \\ ${ }^{8}$ Laboratoire L. Néel—CNRS, BP 138, 38274 Grenoble, France
}

\begin{abstract}
Ferromagnetic metal rings of nanometre range widths and thicknesses exhibit fundamentally new spin states, switching behaviour and spin dynamics, which can be precisely controlled via geometry, material composition and applied f eld. Following the discovery of the 'onion state', which mediates the switching to and between vortex states, a range of fascinating phenomena has been found in these structures. In this overview of our work on ring elements, we f rst show how the geometric parameters of ring elements determine the exact equilibrium spin conf guration of the domain walls of rings in the onion state, and we show how such behaviour can be understood as the result of the competition between the exchange and magnetostatic energy terms. Electron transport provides an extremely sensitive probe of the presence, spatial location and motion of domain walls, which determine the magnetic state in individual rings, while magneto-optical measurements with high spatial resolution can be used to probe the switching behaviour of ring structures with very high sensitivity. We illustrate how the ring geometry has been used for the study of a wide variety of magnetic phenomena, including the displacement of domain walls by electric currents, magnetoresistance, the strength of the pinning potential introduced by nanometre size constrictions, the effect of thermal excitations on the equilibrium state and the stochastic nature of switching events.
\end{abstract}

9 Present address: Applied Physics, Yale University, New Haven, CT 06520, USA. 


\section{Introduction}

The last decade has seen tremendous progress in our understanding of magnetism at the mesoand nanoscale, motivated by the search for new physical phenomena and by the potential for applications. This has been stimulated and made possible by advances in fi $\mathrm{m}$ growth and device fabrication techniques, by the continuous development of new measurement methods and by the tremendous increase in computing power that has allowed the calculation of materials properties at the nano- and microscale. In fact, concurrent progress in these various branches of science and technology has made possible a comprehensive understanding of the properties of materials at the nanoscale, and in particular of small magnetic elements, in such a short time span [1-5].

In this body of work, the ring geometry has attracted much attention by virtue of the fact that it exhibits simple and highly symmetric equilibrium states at remanence, the vortex and the 'onion' states, both existing with mirror equivalents [6]: the vortex state corresponding to a state of circular magnetization (such as that found in disc elements but without the energetically costly vortex core) and the onion state corresponding to the state closest to saturation that is compatible with the topology of the element, i.e., with two halves of the ring magnetization each adopting opposite circulations, each half separated by $180^{\circ}$ domain walls (see insets to f gure 1) $[7,8]$. The character of this domain wall depends on the geometrical parameters of the ring, a consequence of the f ne balance between the exchange and magnetostatic energy terms $[9,10]$. In fact, one could say that for micrometre and submicrometre elements the main term determining the accessible magnetic conf gurations is the magnetostatic energy term, which is intimately linked to the global shape of the element. The fact that the physical boundary is a large portion of the overall system has the effect of severely limiting the number of permissible magnetic states, leading often to the formation of simple equilibrium magnetic conf gurations that reflec the symmetry of the element.

An important property of the ring geometry is that, for a wide range of geometrical parameters, both the onion and vortex states are accessible during the magnetization process and both are stable at remanence $[6,11]$. A typical $M-H$ loop of an array of micrometre size magnetic ring elements is presented in f gure 1. The use of an array of elements enhances the magnetic signal and allows one to assess the spread in the properties of the individual structures, such as switching feld distributions $[12,13]$. Starting from positive saturation, the magnetization decreases slightly, down to zero feld; the resulting state is the onion state, characterized by a large total magnetization. As the magnetic feld is reversed, a jump in the magnetization to a state close to zero remanence is observed, corresponding to the transition from the onion to the vortex state. We will discuss later the reversal mechanism underpinning these transitions. This is followed by another jump, corresponding to the transition from vortex to reverse onion state, and at larger reverse f elds the magnetization increases gently, until the saturated state with all spins parallel to the applied magnetic $\mathrm{f}$ eld is attained. A brief inspection of this $M-H$ curve suggests several questions that one must address, concerning

(i) the range of stability of these magnetic states in terms of the geometrical parameters and material characteristics;

(ii) the mechanisms determining the switching between these different states;

(iii) the character of the domain walls present in the onion state and in particular how they can be used to study domain wall dynamics, domain wall pinning and electron transport properties;

(iv) how to control the switching between any two of the four stable magnetic states and

(v) the magnetic behaviour of more complicated multilayer structures, such as spin-valve systems (multilayer structures exhibiting giant magnetoresistance are desirable for devices). 


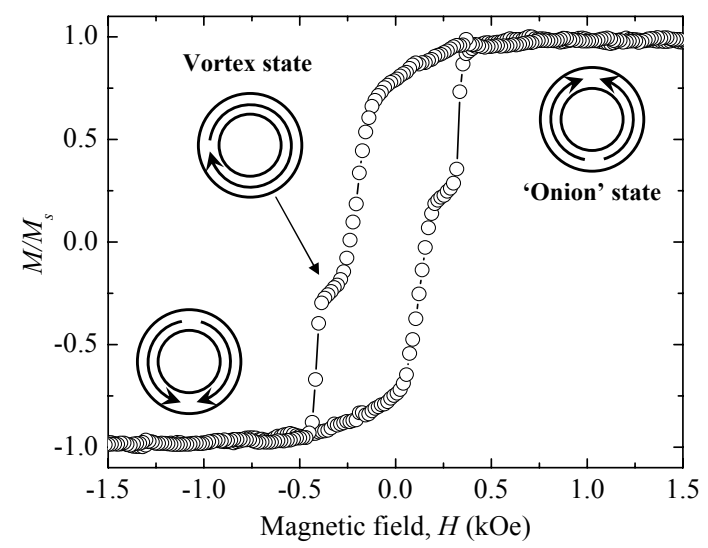

Figure 1. $M-H$ loop of an array of polycrystalline Co rings, $1.65 \mu \mathrm{m}$ outer diameter, $350 \mathrm{~nm}$ wide, $16 \mathrm{~nm}$ thick. The insets show schematic diagrams of the onion and vortex states which are attained during the magnetization reversal process.

Our aim here is to brief y review our work on magnetic ring elements and to demonstrate the attractiveness of this particular geometry for the study of a wide range of topics in magnetism, such as the energetics of equilibrium states, geometrically constrained domain walls, potential wells formed by constrictions, thermal stability of magnetic states, magnetoresistance studies, spin dynamics and current induced domain wall propagation.

\section{Experimental details}

Before discussing the magnetic properties of the ring elements, we consider frst some experimental aspects associated with the fabrication and measurement of this type of structure. This is important, since the fabrication steps determine to a large extent some of the magnetic characteristics of small elements, given the sensitivity of magnetism to structural imperfections and to the presence of impurities; also, the measurement of such small structures often requires that stringent conditions are satisf ed, such as surface smoothness for magneto-optical ref ection measurements, or adequate substrate support in the case of electron transmission measurement techniques $[14,15]$.

Several methods have been developed for the fabrication of small structures and ring elements in particular [8], and in f gure 2 we illustrate some of the most common. One method consists of patterning the continuous magnetic $\mathrm{flm}$ down to the desired shape by means of masking and etching steps (f gure 2, left). It has the advantage that the $\mathrm{flms}$ are grown prior to any processing, allowing for greater control of the $\mathrm{flm}$ microstructure, and the disadvantage that the etching step (typically ion-milling or wet-etching) has been shown to degrade the magnetic properties of patterned features with deep sub-micrometre lateral dimensions [16, 17]. A second method consists of transferring the pattern onto the substrate, followed by $\mathrm{f} / \mathrm{m}$ deposition (f gure 2, middle). This method is particularly useful if semiconductor substrates are used, since the etching of such materials is now well developed down to the nanometre scale [18]. The main disadvantage is that a background magnetic signal is present on the trenches, which may render the measurement process more diffic 1t. A third method consists of depositing a metal $\mathrm{f} 1 \mathrm{~m}$ onto the patterned resist, followed by the removal of unwanted metal and resist in a f nal lift-off step (f gure 2, right). This is a very versatile method, which in principle is material independent and for which the ultimate spatial resolution is determined 


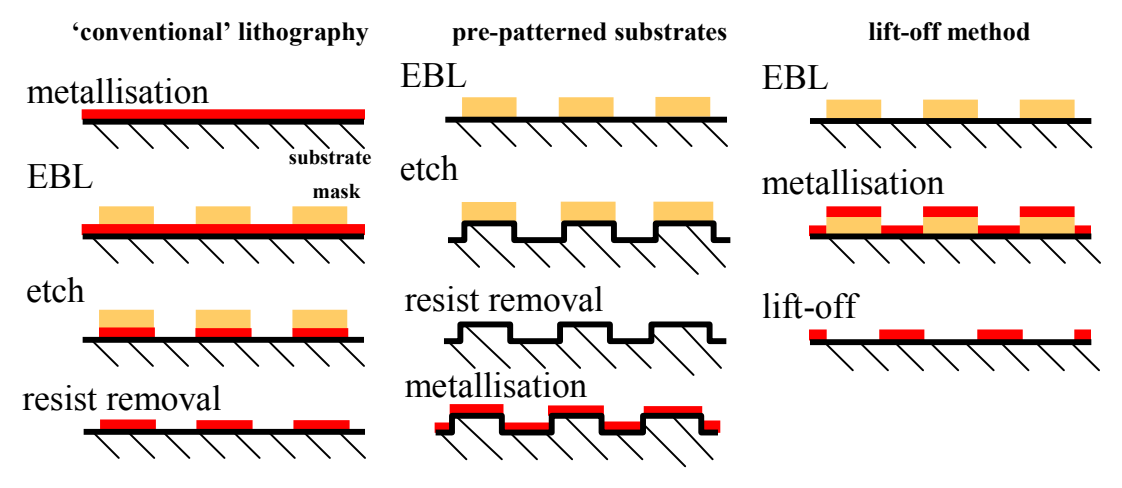

Figure 2. Schematic diagram of three techniques used for the fabrication of thin $\mathrm{f} l \mathrm{~m}$ small elements: 'conventional' lithography (left), use of pre-patterned substrates (middle) and use of PMMA masked substrates or 'lift-off method' (right). (EBL: electron beam lithography.)

by the mask writing step. For electron-beam lithography (EBL) the spatial resolution can be as low as $\sim 10 \mathrm{~nm}$ and the resulting mask is ultrahigh vacuum (UHV) compatible, so that high quality magnetic systems deposited in UHV conditions can be fabricated (as long as no annealing steps are required). The latter method has been adopted for the fabrication of most of our structures, although the use of pre-patterned Si substrates also proved very useful $[6,19]$. Other methods have been developed for the fabrication of small planar structures, such as ion beam irradiation $[20,21]$ and the use of polymer beads as masks for the fabrication of ring elements $[22,23]$.

The $\mathrm{flm}$ growth is another important step in device fabrication, since it bears on the f $1 \mathrm{~m}$ quality (purity, stoichiometry, surface roughness) and crystallinity (defects, crystallite size, grain boundaries, mosaicity or texture). Most structures considered here were grown in UHV in a metal MBE system with a base pressure of the order of $3 \times 10^{-10}$ mbar. For the growth of fcc Co epitaxial samples the Si natural oxide layer is f rst removed by etching in a $10 \%$ HF solution before loading into the load-lock chamber; subsequently, a thick $\mathrm{Cu}$ buffer layer $(60-75 \mathrm{~nm}$ thick) is grown on $\mathrm{Si}(001)$ in order to provide a $\mathrm{Cu}(001)$ template surface for the subsequent growth of epitaxial fcc $\mathrm{Co}(001) \mathrm{flms}$. Otherwise, the ferromagnetic metal $\mathrm{flm}$ is deposited directly onto the $\mathrm{SiO}_{x}$ native layer to provide polycrystalline $\mathrm{flms}$ of $\mathrm{Co}$, $\mathrm{NiFe}$, etc. The latter have the property that no overall magnetocrystalline anisotropy is present. The $\mathrm{NiFe} / \mathrm{Cu} / \mathrm{Co}$ pseudo-spin-valve (PSV) structures considered here were grown using triode sputtering or electron-beam evaporation in systems with base pressures below $5 \times 10^{-9}$ and $9 \times 10^{-8}$ mbar, respectively. Using both metallization techniques on $\operatorname{Si}(100)$ wafers with $50 \mathrm{~nm}$ thick thermal oxide yielded PSV f lms with grain sizes $\sim 10 \mathrm{~nm}$ and giant magnetoresistance ratios up to $1.4 \%$.

We have employed a wide range of measurement techniques for the structural and magnetic characterization of the ring elements, including scanning electron microscopy (SEM), transmission electron microscopy, magneto-optic Kerr effect (MOKE) magnetometry, magnetoresistance (MR) and photoemission electron microscopy (PEEM), among others. For some of these techniques special sample preparation procedures are necessary, but we refer the reader to the original publications for details.

\section{Energetics of equilibrium states}

The equilibrium magnetic conf guration of small magnetic elements is determined by the interplay between the different magnetic energy terms, namely exchange, magnetostatic 

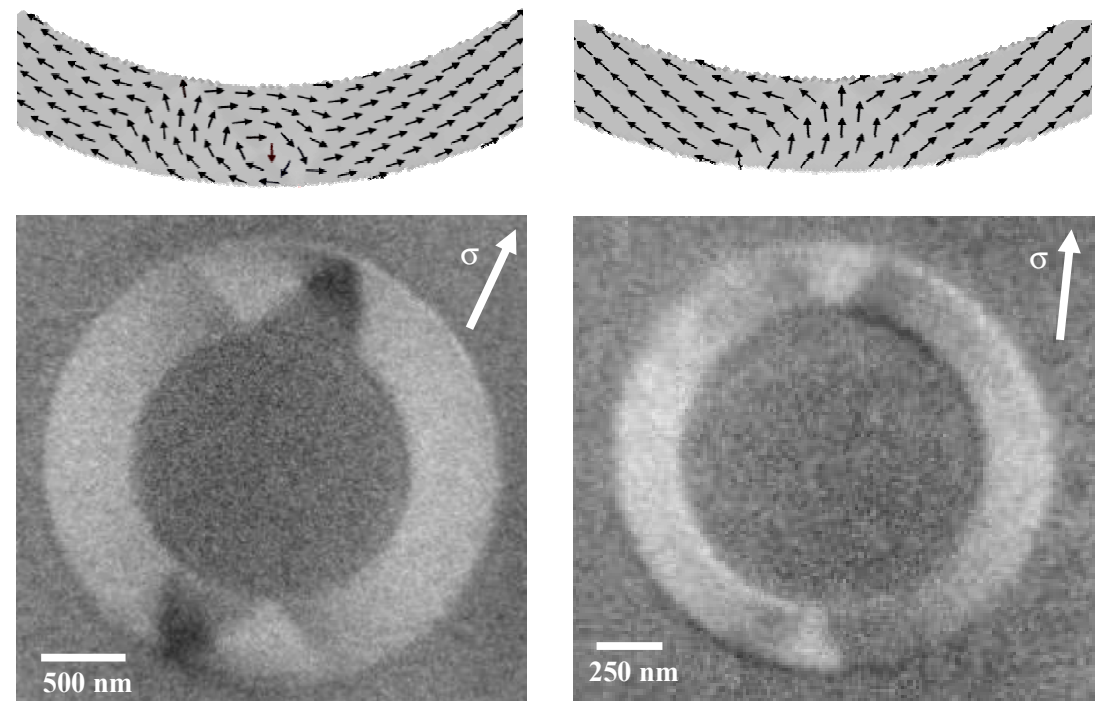

Figure 3. Photoemission electron microscopy (PEEM) images of polycrystalline $\mathrm{Ni}_{80} \mathrm{Fe}_{20}$ rings in the onion state (left, $2.7 \mu \mathrm{m}$ diameter, $530 \mathrm{~nm}$ wide, $30 \mathrm{~nm}$ thick; right, $1.64 \mu \mathrm{m}$ diameter, $260 \mathrm{~nm}$ wide, $10 \mathrm{~nm}$ thick). The direction of magnetic contrast is shown as white arrows $(\sigma$ indicates the in-plane direction of the light polarization). The insets at the top are results of micromagnetic simulations showing the magnetization vector distribution.

and anisotropy terms. While in bulk systems the number of available states tends to be large, as the size is reduced the separation in energy between the different magnetic states becomes progressively larger, leading to the stabilization of a few relatively simple magnetic conf gurations that tend to ref ect the geometrical symmetry of the element. For example, mesoscopic square elements tend to fall into the quadrant, or Landau-Lifshitz, state [24-27], while discs tend to fall into the vortex state [19, 28-30], even though other high symmetry states are accessible in these geometries, such as the diamond and triangle state in disc elements [30-34] or other less symmetric states in squares [35, 36].

As mentioned previously, micrometre size magnetic rings fall into two distinct magnetic states during the magnetization process. While these were originally inferred from MOKE magnetometry and from micromagnetic simulations [6, 37], they were subsequently imaged using scanning electron microscopy with polarization analysis (SEMPA) and photoemission electron microscopy (PEEM) [38]. The spin structure of the $180^{\circ}$ domain walls was also determined, and found to be identical to those found by micromagnetic simulations in straight wires $[39,40]$ : while narrow rings exhibit transverse walls (with a fairly uniformly magnetized triangular region separated by sharper $90^{\circ}$ domain walls), wider rings exhibit vortex walls (consisting of a full magnetization vortex); see f gure 3 .

The existence of these two types of domain walls is a consequence of the competition between the magnetostatic and exchange energy terms: while the exchange energy favours states of uniform magnetization (and hence transverse walls), the magnetostatic energy favours states of fux closure (such as the vortex wall); for narrow rings the exchange energy cost associated with a magnetization vortex precludes such a type of wall and transverse walls are favoured. This is one example of a so-called geometrically constrained domain wall [19, 41-45]. Phase diagrams determining the boundary between these two types of walls in ring elements in the onion state (after saturation by an applied f eld) have been determined experimentally for $\mathrm{Co}$ and $\mathrm{NiFe}$ over a wide range of diameters, widths and thicknesses $[9,10]$. 


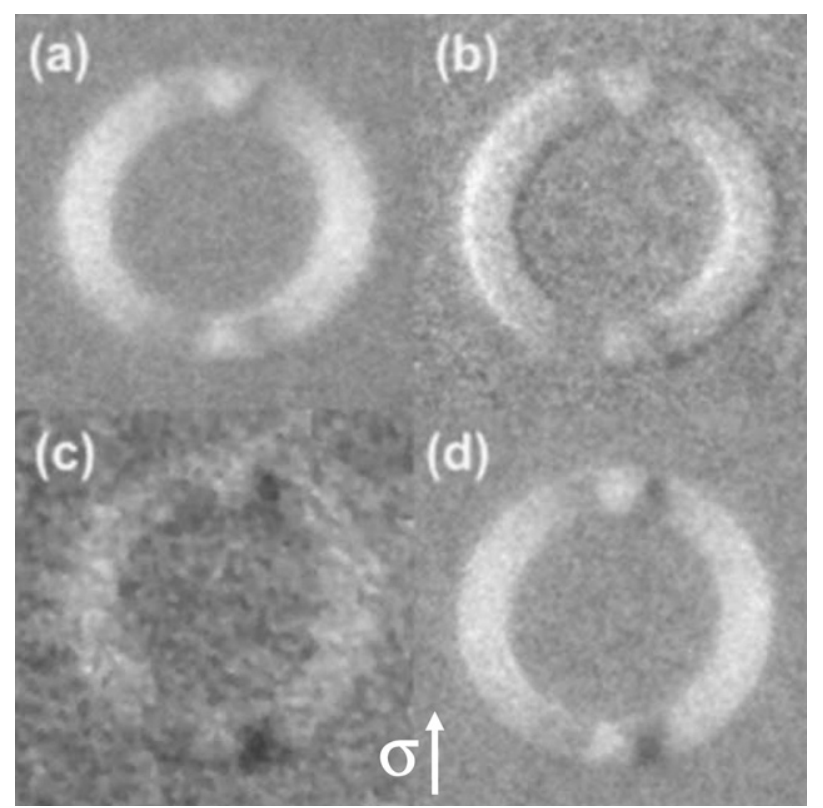

Figure 4. PEEM images showing a thermally activated domain wall transformation in a polycrystalline NiFe ring, $5 \mu \mathrm{m}$ diameter, $730 \mathrm{~nm}$ wide, $7 \mathrm{~nm}$ thick. The images correspond to the same ring, imaged at (a) RT immediately after magnetic saturation, (b) $260^{\circ} \mathrm{C}$, (c) $310^{\circ} \mathrm{C}$ and (d) back at RT. Note the decrease in magnetic contrast with increasing temperature due to the drop in magnetization as the temperature approaches the Curie temperature $\left(T_{\mathrm{c}}=843 \mathrm{~K}\right.$ for bulk $\mathrm{NiFe}[46])$. (From [10].)

It is worth noting that these diagrams do not yield the domain wall corresponding to the lowest energy state, but rather the wall that is stabilized upon removal of the applied magnetic feld. That such a domain wall may not correspond to that of lowest energy has been demonstrated by temperature stability studies of the onion state in NiFe rings lying at the boundary separating rings with vortex or transverse walls [10], f gure 4. In this study, the magnetic ring was f rst saturated by a strong magnetic f eld (4 kOe) at room temperature (RT), such that it exhibited a transverse wall at remanence. Magnetic images of the same ring were obtained for increasing values of the temperature. We fnd that while at $260^{\circ} \mathrm{C}$ the domain wall is still a transverse wall (f gure $4(\mathrm{~b})$ ), at $310^{\circ} \mathrm{C}$ it has transformed into a vortex wall (f gure 4(c)), which remains when the temperature is reduced to RT (f gure 4(d)). This shows that for these particular ring parameters the onion state with the lowest energy corresponds to that with vortex walls, but that an energy barrier effectively stabilizes the transverse wall following magnetic saturation. From the temperature at which this barrier is overcome we estimate this barrier height to be of the order of $50 \mathrm{meV}$ [10].

For the low anisotropy $3 \mathrm{~d}$ transition metal ferromagnets, the effect of magnetic anisotropy on the magnetic behaviour of ring elements is less pronounced than that of the exchange and magnetostatic energy terms. When present, it tends to introduce local magnetic domains that point along the easy axis direction [38] or to introduce a slight anisotropy in the switching behaviour, with the particularity that, in fcc $\mathrm{Co}(001)$ rings, the global easy axis is along the magnetic hard axis of fcc $\mathrm{Co}(001)[47,48]$.

Another possible contribution to the magnetic energy of small in-plane magnetized elements arises from edge imperfections or irregularities that are inevitably present in small 
structures [21, 49-53], either as a consequence of the spatial resolution of the pattern writing or due to the different steps involved during the lithography process or, in the case of structures fabricated by deposition using a pre-def ned mask, by mask irregularities or even due to the size of the crystallites in the case of polycrystalline elements [54]. Numerical calculations suggest that the roughness contribution to the magnetic energy is sizeable for the vortex state (and also by implication for the onion state), is magnetostatic in origin and its effect is to increase the energy of the vortex state [55]. However, as far as energetics is concerned, roughness affects the relative stability of the vortex state vis-à-vis other magnetic states, such as the uniform state. The problem of determining the relative stability of the vortex and onion state in rings is an important one; micromagnetic simulations and experimental observations suggest that the range of stability of these states extends over a large range of parameter values, with outer diameters from $200 \mathrm{~nm}$ to $5 \mu \mathrm{m}$, depending on the ring width and to a lesser extent on the thickness $[8,55,56]$. Roughness plays a more visible role in small elements in determining the nucleation feld for magnetization reversal [12, 49, 54, 57-62], the dynamics of the magnetic reversal [63] and the stabilization of metastable equilibrium states [6, 9, 64].

\section{Field induced magnetic switching}

When considering magnetization dynamics, it is useful to distinguish between the quasi-static and the dynamic regime, corresponding to the cases where the applied magnetic feld varies much more slowly and, respectively, at timescales comparable with or faster than the spin dynamics (of the order of the attempt frequency, $\tau \sim 1 \mathrm{~ns}$ ). In the quasi-static regime the magnetization conf guration corresponds to the minimum in free energy for any given value of the external magnetic feld and one could in principle neglect the magnetization dynamics associated with changes in the magnetic fie d. However, there are instances where this picture breaks down. The presence of several energy minima may result in many possible paths for the magnetization as a function of the magnetic feld in a way that cannot be predicted by simple calculation of the energy minima; in other words, the magnetic state of the sample is history dependent and the equilibrium magnetic state depends on the previous applied feld. Of particular interest in this context are the critical $f$ eld values corresponding to nucleation or reversal of the magnetization, and the particular mechanism which describes the magnetization reversal, namely, coherent rotation, nucleation and domain wall propagation, and nucleation plus domain growth. The switching time and switching f eld amplitude depend sensitively on the particular reversal mechanism favoured by the magnetic system, which is determined by a combination of intrinsic (material properties and shape) and extrinsic (defects, impurities, grain boundaries) factors.

While in f gure 1 the $M-H$ loop exhibits two transitions, one also fnds rings that, for certain values of the geometrical parameters, present a single transition (from onion to reverse onion) and rings with triple switching (in very wide rings, where the switching from the onion to vortex is mediated by the nucleation of an entire magnetic vortex) $[38,65]$. But the essential features of the magnetic switching in ring elements can be understood with reference to rings with double switching, from onion to vortex and vortex to reverse onion. These are representative of two types of magnetic reversal that can occur in small elements: domain wall propagation, and domain nucleation plus wall propagation. In the transition from onion to vortex state one of the $180^{\circ}$ domain walls is displaced and propagates along one half of the ring (annihilating the other domain wall or else forming metastable $360^{\circ}$ domain walls, depending on the relative winding [66]). Which of the walls is displaced and in which direction, and consequently which vortex circulation results, depends on the local pinning potential, and recent experimental results indicate that this process is governed by a stochastic mechanism 
due to thermal fuctuations [67]. In particular, by controlling the local pinning through the introduction of notches, the circulation of the vortex state can be controlled $[68,69]$. The second example occurs in the transition from vortex to onion state; in this case, a reverse domain is $\mathrm{frst}$ nucleated in the half ring with the magnetization opposed to the magnetic f eld, and the two domain walls thus formed propagate until they reach the other half of the ring, forming the two $180^{\circ}$ domain walls of the onion state [6]. Both transitions have been studied numerically and experimentally as a function of the geometrical parameters of the ring (thickness, width and outer diameter) $[56,70]$. It is found that the onion to vortex switching feld depends strongly on the ring width, but less so on the ring diameter and thickness [56, 71]. This can be understood by noting that what determines the domain wall displacement in the onion to vortex transition is the strength of pinning, which arises mostly from edge imperfections; the narrower the ring, the larger the effect of edge irregularities to the pinning of the domain wall and hence the larger the onion to vortex switching f eld. In the case of the vortex to onion transition, a large dependence on the ring width and $\mathrm{f} / \mathrm{m}$ thickness is found [70]. In this situation, the nucleation of the reverse domain depends on the energy balance between Zeeman, magnetostatic and exchange energy terms; generally speaking, the thicker and narrower the $\mathrm{flm}$ the more stable is the vortex state and therefore the larger we expect the switching f eld for the vortex to onion transition to be.

The magnetic switching properties of ring elements have also been studied by magnetoresistance measurements. In these studies, the domain wall structure is probed using the anisotropic magnetoresistance effect (which corresponds to the difference in electron scattering for electric current perpendicular and parallel to the magnetization); one advantage of using magnetoresistance as a magnetic probe is that the signal does not decrease with decreasing element size, making it possible to make single-element measurements. From these studies we can determine the pinning potentials associated with constrictions; in particular, we have shown that a constriction constitutes an attractive potential for transverse walls while it constitutes a repulsive potential for vortex walls [7, 42, 45, 72].

For the situation when the applied magnetic feld varies on timescales comparable to those characteristic of the spin dynamics, the study of the time evolution of the magnetization requires solving the equation of motion of the magnetization as given by the Landau-LifshitzGilbert (LLG) equation (in order to obtain parameter estimates from the experimental data, for instance). Much progress has been accomplished recently in accessing both the spin-wave modes in conf ned geometries [73-78] and in probing the out of equilibrium magnetization dynamics [79-83]. For the specif c case of the ring geometry, ferromagnetic resonance (FMR) [78, 84, 85], time-resolved MOKE [85] and Brillouin light scattering (BLS) [86] measurements have been performed to probe and determine the spin-wave eigenmodes characteristic of magnetic rings. It is observed that, in agreement with what is observed in other small structures, the spin-wave spectra show signatures of the spatial conf nement of the spin-waves, and exhibit modes that conform to the topological geometry of the element (radial and azimuthal modes).

\section{Current induced magnetic switching}

One topic of much current interest is that of current induced magnetic switching (CIMS), whereby the magnetization of a free layer in a spin-valve type of structure or the domain wall in a uniform magnetic medium switches or is displaced by means of a spin polarized electron current (usually polarized by the magnetic medium itself) [53, 87-92]. It holds much promise for the magnetic switching of magnetic memory elements, with the advantage of much simplif ed wiring and writing procedures as compared to conventional magnetic $f$ eld switching. Although it requires very high current densities (of the order of $10^{12} \mathrm{~A} \mathrm{~m}^{-2}$ ), these are applied 


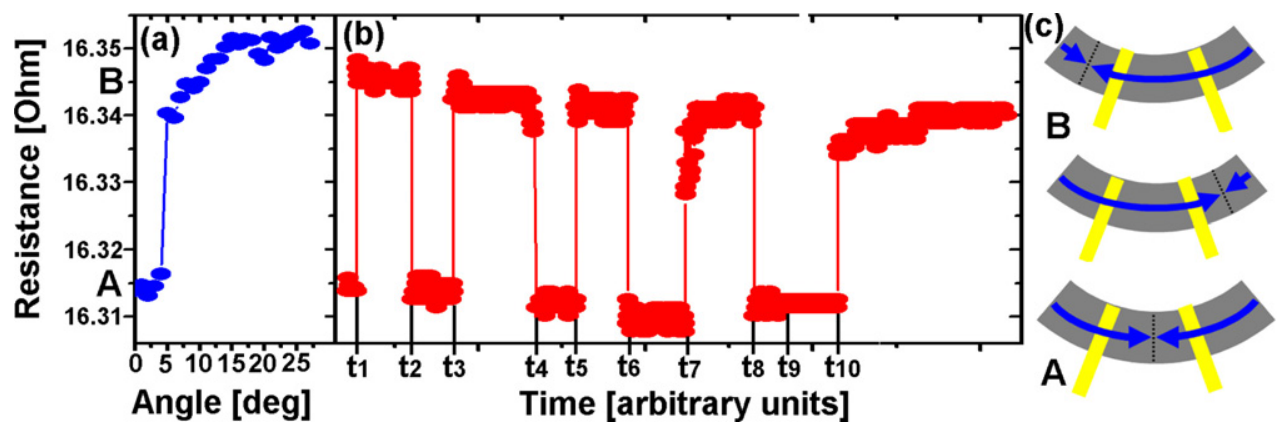

Figure 5. (a) Resistance response as a function of angular position, after the wall is moved by an applied magnetic feld; level A corresponds to the presence of the domain wall in between the voltage contacts, while for level B the domain wall is outside the voltage contacts (for these measurements, the ring is severed at one point so that there is no contribution from other portions of the ring). (b) Resistance across the voltage contacts after successive positive and negative current pulses $\left(20 \mu\right.$ s width, $5 \times 10^{12} \mathrm{~A} \mathrm{~m}^{-2}$ amplitude), demonstrating current induced domain wall motion. (c) Schematic diagrams of the voltage contacts and domain wall position. (From [53].)

in short pulses so that damage to the device is minimized. The ring geometry has proven to be a very useful geometry for the study of current induced domain wall propagation phenomena, since, unlike straight wires, it allows the domain wall to be placed in any desired position by means of a static applied feld. The position of the domain wall can be monitored using magnetoresistance measurements, allowing systematic studies of the critical current for domain wall displacement as a function of the geometrical and material parameters $[10,53,93,94]$, or to study the temperature dependence of the domain wall depinning and displacement [95]. In f gure 5 we present the results of experiments demonstrating current induced domain wall motion in single layer permalloy rings. Here, the domain wall movement can be detected by the drop in resistance due to the AMR (f gure 5(a)). After positioning the domain wall just outside the voltage contacts, a current pulse is applied displacing the domain wall into the region limited by the voltage contacts, lowering the resistance; successive pulses can move the wall entirely out (past the second voltage contact) and reverse current pulses are able to move the wall back.

The experiments performed in this geometry, and variants thereof, have demonstrated the sensitivity of the spin-torque effect to the particular spin structure, a conclusion that has obvious important practical implications; they also show that repeated current pulses tend to distort the domain wall, and restoration mechanisms must be envisaged in order to ensure repeatability of the current induced switching process $[40,53,96]$. However, many issues remain open, such as optimization of the spin-torque eff ciency, for instance by considering high spin-polarization materials or multilayer structures, topics which are currently under investigation.

\section{Magnetic properties of spin-valve ring elements}

One natural extension of the work on ring elements is to consider multilayer ring structures exhibiting giant magnetoresistance (GMR), such as spin-valves [97, 98]. Pseudo-spinvalve ring structures displaying GMR have been fabricated and their magnetization reversal investigated using magnetoresistance measurements and micromagnetic modelling [99-102]. These structures consist of a soft (free) layer $(\mathrm{NiFe})$ and a hard layer $(\mathrm{Co})$ separated by a non-magnetic $\mathrm{Cu}$ layer, suff ciently thick to largely decouple the two magnetic layers (such 

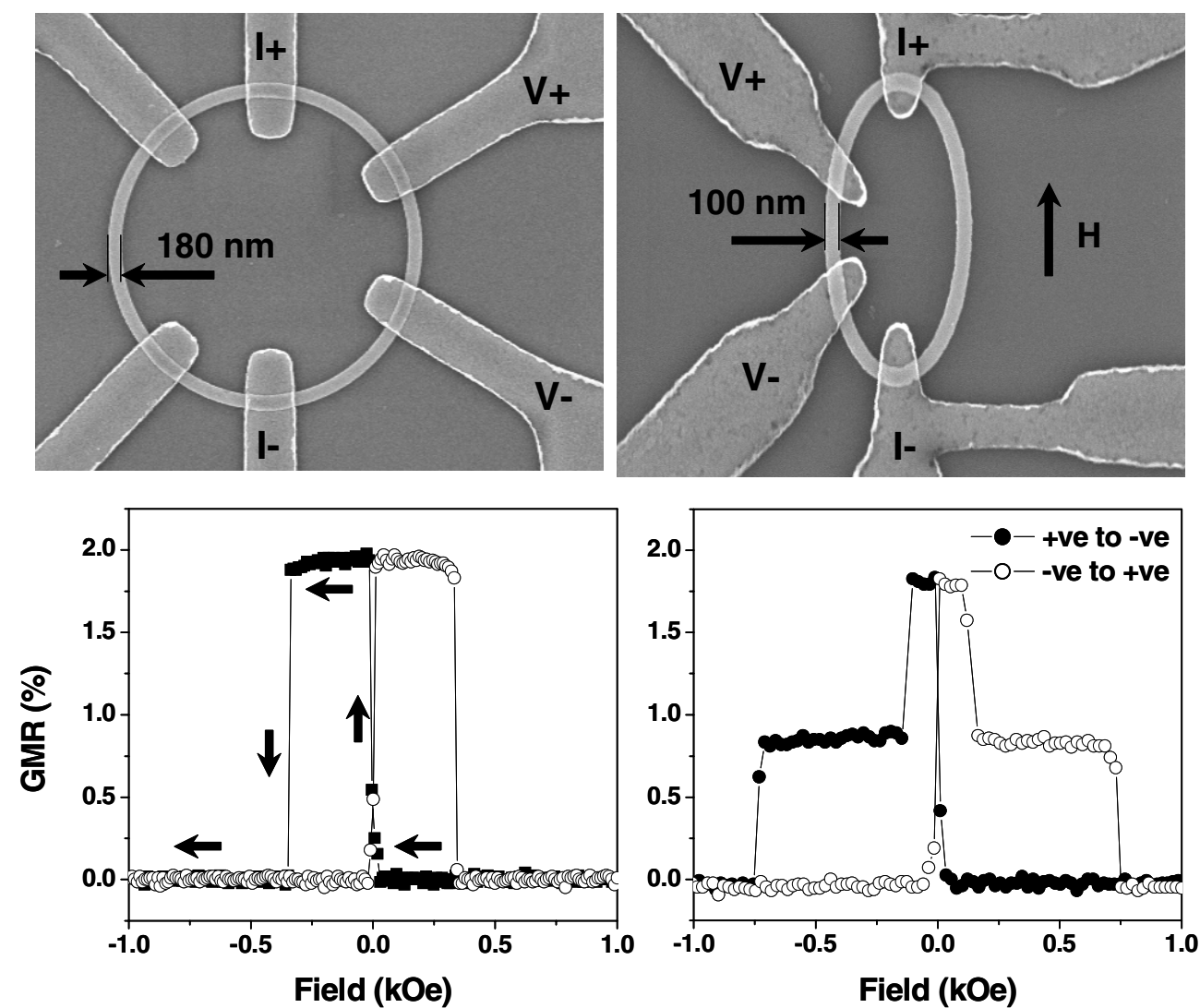

Figure 6. Top: scanning electron microscopy (SEM) of pseudo-spin-valve rings fabricated used electron-beam lithography. Bottom: respective magnetoresistive characteristics for the contact geometries indicated in the SEM image, corresponding to four-point contact measurements. The ring outer diameter and the major axis of the elliptical ring are $5 \mu \mathrm{m}$ and the magnetic fiel is applied in the direction of the current contacts.

that interlayer exchange coupling effects are negligible). However, due to the magnetostatic coupling between the two magnetic layers (due to the stray f eld associated with the onion state), the switching properties of the soft layer are strongly affected by the stray f eld issuing from the Co layer (while the behaviour of the Co layer remains largely unaffected) [99, 100, 102].

Figure 6 shows examples of the magnetoresistive behaviour of pseudo-spin-valve rings, $5 \mu \mathrm{m}$ wide, with nominal structure $\mathrm{Au}(4 \mathrm{~nm}) / \mathrm{Co}(7 \mathrm{~nm}) / \mathrm{Cu}(5 \mathrm{~nm}) / \mathrm{NiFe}(4 \mathrm{~nm}) / \mathrm{SiO}_{x} / \mathrm{Si}(001)$, for two types of ring geometries studied, circular and elliptical. The data for the circular ring show a f at baseline interrupted by abrupt jumps as domain walls are created and move through the ring; this leads to an increase in resistance as the parallel arrangement of the magnetization of the $\mathrm{NiFe}$ and $\mathrm{Co}$ (both in the same onion state) changes to an anti-parallel spin arrangement following the reversal of the NiFe layer to the reverse onion state. For the elliptical ring, the magnetoresistance data shows a fat baseline and very abrupt transitions as the domain walls in the NiFe layer sweep through the region limited by the voltage contacts. As the reverse f eld increases, the propagating domain walls in the NiFe meet, forming a $360^{\circ}$ wall on each side of the elliptical ring, similar to the $360^{\circ}$ walls reported in small circular rings ('twistedonion' state) $[66,98,103]$; the $360^{\circ}$ walls have an insignif cant effect on the GMR, and since 
the Co layer is still in the onion state, the resistance of the ring is maximum. The intermediate resistance level corresponds to the switching of the Co layer to the vortex state and the transition to the reverse onion state results again in a minimum resistance value [99]. This interpretation of the experimental results is supported by three-dimensional micromagnetic simulations of both the magnetization and of the GMR amplitude $[99,100]$. Also clear from fi ure 6 is that the elliptical geometry offers increased stability of the vortex state in the hard layer. Recent experimental results indicate that the switching processes in these types of spin-valve structures are strongly dependent on the amplitude of the saturating applied f eld and that the fi e structure of the reversal process (which can be accessed by measuring minor hysteresis loops) is essentially controlled by the hard Co layer $[102,104]$.

\section{Conclusions}

In conclusion, ferromagnetic rings of nanometre range widths and thicknesses exhibit fundamentally new spin states, switching behaviour and spin dynamics which can be precisely controlled via geometry, material composition and applied feld. This precise control of the magnetic ring characteristics makes it a very attractive geometry for devices, including MRAM and magnetic sensors $[97,98,105,106]$. It also makes the ring geometry particularly suited for the study of a range of magnetic phenomena, as this overview illustrates with examples extracted from our work.

\section{Acknowledgments}

The authors thank the participants of the ESF workshop 'Mott's Physics in Nanowires and Quantum Dots' (Cambridge, UK, 31 July-2 August, 2006) for discussions, and the European Science Foundation (ESF) for support. This work has also been supported by the EPSRC (UK), the CMI Magnetoelectronic Devices project and the Deutsche Forschungsgemeinschaft (SFB 513). Part of this work was performed at the SLS, Paul Scherrer Institut, Villigen, Switzerland and at Elettra, Trieste, Italy.

\section{References}

[1] Dennis C L, Borges R P, Buda L D, Ebels U, Gregg J F, Hehn M, Jouguelet E, Ounadjela K, Petej I, Prejbeaunu I L and Thornton M J 2002 J. Phys.: Condens. Matter 14 R1175

[2] Hillebrands B and Ounadjela K (ed) 2002 Spin Dynamics in Confin d Magnetic Structures I (Springer Topics in Applied Physics vol 83) (Berlin: Springer)

[3] Hillebrands B and Ounadjela K (ed) 2003 Spin Dynamics in Confin d Magnetic Structures II (Springer Topics in Applied Physics vol 87) (Berlin: Springer)

[4] Martín J I, Nogués J, Liu K, Vicent J L and Schuller I K 2003 J. Magn. Magn. Mater. 256449

[5] Mills D L and Bland J A C (ed) 2006 Nanomagnetism (Contemporary Concepts of Condensed Matter Science) (Amsterdam: Elsevier)

[6] Rothman J, Kläui M, Lopez-Diaz L, Vaz C A F, Bleloch A, Bland J A C, Cui Z and Speaks R 2001 Phys. Rev. Lett. 861098

[7] Kläui M, Vaz C A F, Bland J A C, Wernsdorfer W, Faini G and Cambril E 2003 J. Appl. Phys. 937885

[8] Kläui M, Vaz C A F, Lopez-Diaz L and Bland J A C 2003 J. Phys.: Condens. Matter 15 R985

[9] Kläui M, Vaz C A F, Bland J A C, Heyderman L J, Nolting F, Pavlovska A, Bauer E, Cherif S, Heun S and Locatelli A 2004 Appl. Phys. Lett. 855637

[10] Laufenberg M et al 2006 Appl. Phys. Lett. 88052507

[11] Moore T A, Hayward T J, Tse D H Y, Bland J A C, Castaño F J and Ross C A 2005 J. Appl. Phys. 97063910

[12] Kläui M, Vaz C A F, Bland J A C, Sinnecker E H C P, Guimarães A P, Wernsdorfer W, Faini G, Cambril E, Heyderman L J and David C 2004 Appl. Phys. Lett. 84951 
[13] Kläui M, Vaz C A F, Heyderman L J, Rüdiger U and Bland J A C 2005 J. Magn. Magn. Mater. 290/291 61

[14] Heyderman L J, Kläui M, Schäublin R, Rüdiger U, Vaz C A F, Bland J A C and David C 2005 J. Magn. Magn. Mater. 290/291 86

[15] Backes D et al 2006 Microelectron. Eng. 831726

[16] Castaño F J, Haratani S, Hao Y, Ross C A and Smith H I 2002 Appl. Phys. Lett. 812809

[17] Katine J A, Ho M K, Ju Y S and Rettner C T 2003 Appl. Phys. Lett. 83401

[18] Cui Z, Rothman J, Kläui M, Lopez-Diaz L, Vaz C A F and Bland J A C 2002 Microelectron. Eng. 61/62 577

[19] Vaz C A F, Lopez-Diaz L, Kläui M, Bland J A C, Monchesky T L, Unguris J and Cui Z 2003 Phys. Rev. B $67140405(\mathrm{R})$

[20] Chappert C et al 1998 Science 2801919

[21] Kläui M, Vaz C A F, Lapicki A, Suzuki T, Cui Z and Bland J A C 2004 Microelectron. Eng. 73/74 785

[22] Zhu F Q, Fan D, Zhu X, Zhu J-G, Cammarata R C and Chien C-L 2004 Adv. Mater. 162155

[23] Zhu F Q, Chern G W, Tchernyshyov O, Zhu X C, Zhu J G and Chien C L 2006 Phys. Rev. Lett. 96027205

[24] Kirk K J, Chapman J N and Wilkinson C D W 1997 Appl. Phys. Lett. 71539

[25] Dunin-Borkowski R E, McCartney M R, Kardynal B and Smith D J 1998 J. Appl. Phys. 84374

[26] Cherif S, Hertel R, Kirschner J, Wang H, Belkhou R, Locatelli A, Heun S, Pavlovska A and Bauer E 2005 J. Appl. Phys. 98043901

[27] Heyderman L J, Czekaj S, Nolting F, Müller E, Fischer P, Gasser Ph and López-Díaz L 2005 J. Appl. Phys. 99063904

[28] Cowburn R P, Koltsov D K, Adeyeye A O, Welland M E and Tricker D M 1999 Phys. Rev. Lett. 831042

[29] Schneider M, Hoffmann H and Zweck J 2000 Appl. Phys. Lett. 772909

[30] Zhu J-G and Zheng Y 2002 The micromagnetics of magnetoresistive randon access memory Spin Dynamics in Confin d Magnetic Structures I (Springer Topics in Applied Physics) ed B Hillebrands and K Ounadjiela (Berlin: Springer) p 289

[31] Guslienko K Yu, Novosad V, Otani Y, Shima H and Fukamichi K 2002 Phys. Rev. B 65024414

[32] Prejbeaunu I L, Natali M, Buda L D, Ebels U, Lebib A, Chen Y and Ounadjela K 2002 J. Appl. Phys. 917343

[33] Vaz C A F, Kläui M, Heyderman L J, David C, Nolting F and Bland J A C 2005 Phys. Rev. B 72224426

[34] Vaz C A F, Kläui M, Bland J A C, Heyderman L J, David C and Nolting F 2006 Nucl. Instrum. Methods Phys. Res. B 24613

[35] Gu E, Ahmad E, Gray S J, Daboo C, Bland J A C, Brown L M, Rührig M, McGibbon A J and Chapman J N 1997 Phys. Rev. Lett. 781158

[36] Gubbiotti G, Albani L, Carlotti G, De Crescenzi M, Di Fabrizio E, Gerardino A, Donzelli O, Nizzoli F, Koo H and Gomez R D 2000 J. Appl. Phys. 875633

[37] Li S P, Peyrade D, Natali M, Lebib A, Chen Y, Ebels U, Buda L D and Ounadjela K 2001 Phys. Rev. Lett. 861102

[38] Kläui M et al 2003 Phys. Rev. B 68134426

[39] McMichael R D and Donahue M J 1997 IEEE Trans. Magn. 334167

[40] Nakatani Y, Thiaville A and Miltat J 2005 J. Magn. Magn. Mater. 290/291 750

[41] Bruno P 1999 Phys. Rev. Lett. 832425

[42] Kläui M, Vaz C A F, Rothman J, Bland J A C, Wernsdorfer W, Faini G and Cambril E 2003 Phys. Rev. Lett. 90097202

[43] Vaz C A F, Lopez-Diaz L, Kläui M, Bland J A C, Monchesky T, Unguris J and Cui Z 2004 J. Magn. Magn. Mater. 272-276 1674

[44] Jubert P-O, Allenspach R and Bischof A 2004 Phys. Rev. B 69220410

[45] Kläui M et al 2005 Appl. Phys. Lett. 87102509

[46] Wijn H P J 1991 Magnetic properties of metals: d-elements, alloys and compounds Data in Science and Technology (Berlin: Springer)

[47] Lopez-Diaz L, Rothman J, Kläui M and Bland J A C 2001 J. Appl. Phys. 897579

[48] Vaz C A F, Kläui M, Bland J A C, Heyderman L J and Nolting F 2004 J. Appl. Phys. 956732

[49] Uhlig W C and Shi J 2004 Appl. Phys. Lett. 84759

[50] Yang X, Liu C, Ahner J, Yu J, Klemmer T, Johns E and Weller D 2004 J. Vac. Sci. Technol. B 231

[51] Estève J, Aussibal C, Schumm T, Figl C, Mailly D, Bouchoule I, Westbrook C I and Aspect A 2004 Phys. Rev. A 70043629

[52] Heyderman L J, Kläui M, Nöhammer B, Vaz C A F, Bland J A C and David C 2004 Microelectron. Eng. 73/74 780

[53] Kläui M, Vaz C A F, Bland J A C, Wernsdorfer W, Faini G, Cambril E, Heyderman L J, Nolting F and Rüdiger U 2005 Phys. Rev. Lett. 94106601

[54] Schref T, Fidler J, Kirk K J and Chapman J N 1999 J. Appl. Phys. 856169 
[55] Vaz C A F, Athanasiou C, Bland J A C and Rowlands G 2006 Phys. Rev. B 73054411

[56] Yoo Y G, Kläui M, Vaz C A F, Heyderman L J and Bland J A C 2003 Appl. Phys. Lett. 822470

[57] Gadbois J and Zhu J-G 1995 IEEE Trans. Magn. 313802

[58] Shi J and Tehrani S 2000 Appl. Phys. Lett. 771692

[59] Herrmann M, McVitie S and Chapman J N 2000 J. Appl. Phys. 872994

[60] Suess D, Tsiantos V, Schref T, Scholz W and Fidler J 2002 J. Appl. Phys. 917977

[61] Shima H, Novosad V, Otani Y, Fukamichi K, Kikuchi N, Kitakamai O and Shimada Y 2002 J. Appl. Phys. 921473

[62] Brian M T, Atkinson A and Cowburn R P 2004 Appl. Phys. Lett. 853510

[63] Cayssol F, Ravelosona D, Chappert C, Ferré J and Jamet J P 2004 Phys. Rev. Lett. 92107202

[64] Liou S H, Sabiryanov R F, Jaswal S S, Wu J C and Yao Y D 2001 J. Magn. Magn. Mater. 226-230 1270

[65] Kläui M, Vaz C A F, Bland J A C, Heyderman L J, David C, Sinnecker E H C P and Guimarães A P 2004 J. Appl. Phys. 956639

[66] Castaño F J, Ross C A, Frandsen C, Eilez A, Gil D, Smith H I, Redjdal M and Humphrey F B 2003 Phys. Rev. B 67184425

[67] Hayward T J, Moore T A, Tse D H Y, Bland J A C, Castaño F J and Ross C A 2005 Phys. Rev. B 72184430

[68] Kläui M, Rothman J, Lopez-Diaz L, Vaz C A F, Bland J A C and Cui Z 2001 Appl. Phys. Lett. 783268

[69] Kläui M, Vaz C A F, Bland J A C, Wernsdorfer W, Faini G and Cambril E 2002 Appl. Phys. Lett. 81108

[70] Kläui M, Lopez-Diaz L, Rothman J, Vaz C A F, Bland J A C and Cui Z 2002 J. Magn. Magn. Mater. 2407

[71] Lopez-Diaz L, Rothman J, Kläui M and Bland J A C 2000 IEEE Trans. Magn. 363155

[72] Kläui M, Vaz C A F, Wernsdorfer W, Bauer E, Cherif S, Heun S, Locatelli A, Faini G, Cambril E and Bland J A C 2004 Physica B 343343

[73] Goglio G, Pignard S, Radulescu A, Piraux L, Huynen I, Vanhoenacker D and Vander Vorst A 1999 Appl. Phys. Lett. 751769

[74] Demokritov S O, Hillebrands B and Slavin A N 2001 Phys. Rep. 348441

[75] Gubbiotti G, Carlotti G, Nizzoli F, Zivieri R, Okuno T and Shinjo T 2002 IEEE Trans. Magn. 382532

[76] Demidov V E, Demokritov S O, Hillebrands B, Laufenberg M and Freitas P P 2004 Appl. Phys. Lett. 852866

[77] Martyanov O N, Yudanov V F, Lee R N, Nepijko S A, Elmers H J, Schneider C M and Schönhense G 2005 Appl. Phys. A $\mathbf{8 1} 679$

[78] Giesen F, Podbielski J, Korn T, Steiner M, van Staa A and Grundler D 2005 Appl. Phys. Lett. 86112510

[79] Ballentine G E, Hiebert W K, Stankiewicz A and Freeman M R 2000 J. Appl. Phys. 876830

[80] Acremann Y, Buess M, Back C H, Dumm M, Bayreuther G and Pescia D 2001 Nature 41451

[81] Hicken R J, Hughes N D, Moore J R, Schmool D S, Wilks R and Wu J 2002 J. Magn. Magn. Mater. 242-245 559

[82] Kuksov A, Schneider C M, Oelsner A, Krasyuk A, Neeb D, Schönhense G, De Nadaï C and Brookes N B 2004 J. Appl. Phys. 956530

[83] Choi B C and Freeman M R 2005 Nonequilibrium spin dynamics in laterally def ned magnetic structures Ultrathin Magnetic Structures III ed J A C Bland and B Heinrich (Berlin: Springer) p 211

[84] Giesen F, Podbielski J, Korn T and Grundler D 2005 J. Appl. Phys. 97 10A712

[85] Neudecker I, Kläui M, Perzlmaier K, Backes D, Heyderman L J, Vaz C A F, Bland J A C, Rüdiger U and Back C H 2006 Phys. Rev. Lett. 96057207

[86] Schultheiss H, Nembach H T, Weber M C, Blomeier S, Beck P A, Candeloro P, Bayer C, Leven B, Hillebrands B, Podbielski J, Giesen F and Grundler D 2006 Space resolved dynamics in small magnetic rings, submitted

[87] Berger L 1978 J. Appl. Phys. 492156

[88] Slonczewski J C 1996 J. Magn. Magn. Mater. 159 L1

[89] Katine J A, Albert F J, Buhrman R A, Myers E B and Ralph D C 2000 Phys. Rev. Lett. 843149

[90] Grollier J, Lacour D, Cros V, Hamzic A, Vaurés A, Fert A, Adam D and Faini G 2002 J. Appl. Phys. 924825

[91] Wegrowe J-E, Fábián A, Guittienne Ph, Hoffer X, Kelly D, Ansermet J-Ph and Olive E 2002 Appl. Phys. Lett. 803775

[92] Edwards D M and Mathon J 2006 Current-induced switching of magnetisation Nanomagnetism (Contemporary Concepts of Condensed Matter Science) ed D L Mills and J A C Bland (Amsterdam: Elsevier) p 273

[93] Kläui M, Vaz C A F, Bland J A C, Wernsdorfer W, Faini G, Cambril E and Heyderman L J 2003 Appl. Phys. Lett. 83105

[94] Kläui M et al 2006 Appl. Phys. Lett. 88232507

[95] Laufenberg M, Bührer W, Bedau D, Melchy P-E, Kläui M, Vila L, Faini G, Vaz C A F, Bland J A C and Rüdiger U 2006 Phys. Rev. Lett. 97046602

[96] Kläui M, Jubert P-O, Allenspach R, Bischof A, Bland J A C, Faini G, Rüdiger U, Vaz C A F, Vila L and Vouille C 2005 Phys. Rev. Lett. 95026601 
[97] Zhu J-G, Zheng Y and Prinz G A 2000 J. Appl. Phys. 876668

[98] Zhu X and Zhu J-G 2003 IEEE Trans. Magn. 392854

[99] Castaño F J, Morecroft D, Jung W and Ross C A 2005 Phys. Rev. Lett. 95137201

[100] Morecroft D, Castaño F J, Jung W, Feuchtwanger J and Ross C A 2006 Appl. Phys. Lett. 88172508

[101] Morecroft D, Castaño F J, Jung W and Ross C A 2006 J. Appl. Phys. $9908 \mathrm{~T} 104$

[102] Hayward T J, Llandro J, Bland J A C, Morecroft D, Castaño F J and Ross C A 2006 Phys. Rev. B 74134405

[103] Castaño F J, Ross C A, Eilez A, Jung W and Frandsen C 2004 Phys. Rev. B 69144421

[104] Castaño F J, Morecroft D and Ross C A 2006 Low-f eld giant magnetoresistance in layered magnetic rings Phys. Rev. B 74224401

[105] Miller M M, Prinz G A, Cheng S-F and Bounnak S 2002 Appl. Phys. Lett. 812211

[106] Hayward T J, Llandro J, Balsod R B, Bland J A C, Castaño F J, Morecroft D, Castaño F J and Ross C A 2006 Appl. Phys. Lett. 89112510 